\title{
Prevalence and characteristics of hearing and vision loss in preschool children from low income South African communities: results of a screening program of 10,390 children
}

\author{
Susan Eksteen ${ }^{1^{*}} \mathbb{D}$, Robert H. Eikelboom ${ }^{1,2,3}$, Hannah Kuper ${ }^{4}$, Stefan Launer ${ }^{5,6}$ and De Wet Swanepoel ${ }^{1,2}$
}

\begin{abstract}
Background: The majority of children with sensory impairments live in low- and middle-income countries. More studies of hearing and vision impairment prevalence are needed, in order to generate more accurate estimates of trends in sensory impairments. This study aimed to estimate the prevalence and describe the characteristics of hearing and vision loss among preschool children (4-7years) in an underserved South African community following community-based mobile health (mHealth) supported hearing and vision services.

Methods: A screening program of sensory impairments was undertaken of children attending preschools in the communities of Khayelitsha and Mitchell's Plain, Cape Town, from September 2017 until June 2019. Hearing and vision screening were done by trained community health workers using mHealth technology. Children who failed hearing and vision screening were seen for follow-up assessments at their preschools. Follow-up assessments were conducted using smartphones that host point-of-care validated and calibrated hearing and vision testing applications (hearTest app, hearX Group, South Africa and PeekAcuity app, Peek Vision, United Kingdom). Descriptive statistical analysis and logistic regression analysis were conducted after extracting data from a secure cloud-based server (mHealth Studio, hearX Group) to Microsoft Excel (2016).
\end{abstract}

Results: A total of 10,390 children were screened at 298 preschools over 22 months. Of the children screened, 5.6 and $4.4 \%$ of children failed hearing and vision screening respectively. Community-based follow-up hearing tests were done at the preschools on $88.5 \%$ (514) of children of whom 240 children (54.2\% female) presented with hearing loss. A preschool-based follow-up vision test was done on 400 children (88.1\%). A total of 232 children (46.1\% female) had a vision impairment, and a further 32 children passed the test but had obvious signs of ocular morbidity. Logistic regression analysis found that age was a significant predictor of vision loss $(p<0.05)$, but not for hearing loss $(p=0.06)$. Gender was not a significant predictor of hearing $(p=0.22)$ or vision loss $(p=0.20)$.

Conclusions: Hearing loss is prevalent in at least 22 per 1000 and vision loss in at least 23 per 1000 preschool children in an underserved South African community. Timely identification of sensory losses can be facilitated through community-based hearing and vision services supported by mHealth technology.

Keywords: Hearing loss, Vision loss, Preschool children, Low- and middle-income community, mHealth

\footnotetext{
*Correspondence: susaneksteen17@gmail.com

${ }^{1}$ Department of Speech-Language Pathology and Audiology, University

of Pretoria, Lynnwood Rd, Hatfield, Pretoria, Gauteng, South Africa

Full list of author information is available at the end of the article
}

(c) The Author(s) 2022. Open Access This article is licensed under a Creative Commons Attribution 4.0 International License, which permits use, sharing, adaptation, distribution and reproduction in any medium or format, as long as you give appropriate credit to the original author(s) and the source, provide a link to the Creative Commons licence, and indicate if changes were made. The images or other third party material in this article are included in the article's Creative Commons licence, unless indicated otherwise in a credit line to the material. If material is not included in the article's Creative Commons licence and your intended use is not permitted by statutory regulation or exceeds the permitted use, you will need to obtain permission directly from the copyright holder. To view a copy of this licence, visit http://creativecommons.org/licenses/by/4.0/. The Creative Commons Public Domain Dedication waiver (http://creativeco mmons.org/publicdomain/zero/1.0/) applies to the data made available in this article, unless otherwise stated in a credit line to the data. 


\section{Background}

Childhood hearing and vision loss are significant contributors to the global burden of disease [1,2] affecting 38.7 and 32.5 million children under 10 years, respectively [2]. According to the World Health Organization (WHO), the majority of childhood hearing loss $(60 \%)$ and vision loss $(80 \%)$ can be treated or prevented if identified early [3, 4]. Therefore, periodic hearing and vision screening are considered integral strategies for preventative paediatric health care [5-8]. Early detection of sensory impairments is essential for facilitating early childhood development, socioemotional well-being and academic success, [1, 912] as well as the sustainable development goals (SDGs) related to education $[1,2,13]$. Early-childhood screening in preschools can identify children with congenital sensory losses, as well as those with late-onset, progressive, or fluctuating hearing and vision loss, thus facilitating intervention prior to school entry $[6,12,14-16]$.

Unfortunately, the majority of children (80 to 90\%) with sensory impairments live in low- and middle-income countries (LMICs) $[2,4,10,12]$ where services are usually unavailable or inaccessible, because of an absence of systematic screening programmes for children, prohibitive equipment cost and a shortage of trained personnel $[11,17,18]$. The prevalence of hearing and vision loss for children aged between 5 and 9 years are estimated at 4.5 and $3.1 \%$ respectively in sub-Saharan Africa in contrast to 2.2 and $1.3 \%$ respectively in high-income North America, demonstrating the need for attention to sensory impairment in LMICs [2]. Most cases of childhood hearing and vision loss have preventable causes that are common in low-to-middle-income countries (LMICs) and is often related either to infection or nutrition [8$10,16,19]$. Unfortunately, children with disabilities in LMICs have considerably limited access to non-emergency health resources $[11,19]$ and are therefore prone to be left behind under the SDGs era without timely and appropriate intervention from early childhood $[2,16,20]$.

Estimating the prevalence of sensory loss in this population is an important step to ensure adequate planning and successful implementation of community-based hearing and vision care in preschools in this context. There is a lack of contemporary population-based information about childhood hearing loss and visual impairment, from which the scope and priorities for prevention and treatment can be identified [1, 2, 6, 10, 12, 21, 22]. Particularly in high-burden LMICs, where these disabling conditions are highly prevalent, more studies of hearing and vision impairment prevalence are needed, in order to generate more accurate estimates of trends in sensory impairments $[1,2,10]$. Until recently, these surveys have been complex to undertake, relying on expensive equipment and trained staff, explaining the lack of data. The past few years have seen a rapid expansion of the evidence base on the value of community-based programmes incorporating non-professionals using solutions based on smartphone and internet technologies (mobile health (mHealth) technology) for hearing and vision services [15, 21, 23-30]. A South African study by Eksteen et al., (2019) recently reported the first hearing and vision screening for preschool children using smartphone-based technologies [26]. In this study, trained community health workers (CHWs) used validated smartphone-based applications (apps) for hearing screening (hearScreen app; hearX Group, South Africa) $[21,23,24,26-28,30]$ and vision screening (Peek Acuity app; peekVision, United Kingdom) [15, 25]. In order to overcome loss to follow-up previously shown to affect the outcomes of screening programmes $[8,23,24,31]$, the study included a community-based first-line follow-up assessment for those who failed screening by also utilizing validated mHealth technology [26].

The aim of this study was to estimate the prevalence and describe the characteristics of hearing and vision loss among preschool children (4-7 years) in an underserved South African community following the mHealth supported community-based hearing and vision services described by Eksteen et al., (2019).

\section{Methods}

Institutional Review Board clearance for the study was obtained from the University of Pretoria (HUM020/1019).

\section{Context and population}

A community-based hearing and vision screening program for preschool children by community health workers (CHWs) was implemented using validated mHealth technologies [26, 32]. Four non-professionals from the community, none who had previous training in hearing or vision healthcare, were appointed and trained as CHWs to conduct the hearing and vision screening of all children included in the study at their preschools of the partially informal townships of Khayelitsha and Mitchell's Plain in South Africa [26, 32]. This program was undertaken from September 2017 to June 2019. The majority (97.1\%; (181,145/186803)) of households within Khayelitsha and Mitchell's Plain are classified as low- and middle-income and the population of children aged 5 to 9 years was estimated as 61,094 in 2011 [33]. All children between the ages of 4 and 7 years attending preschools in the targeted areas for whom consent was obtained, received hearing and vision screening tests [26]. Children who failed either test had a follow-up assessment at their preschool [26]. If indicated, children were referred to their nearest clinic for intervention. This study estimated 
the prevalence of hearing and vision loss, based upon the results of the follow-up assessment.

\section{Initial screening for hearing and vision}

Hearing and vision screening were done by trained $\mathrm{CHWs}$ at the preschools in the community using smartphones that host point-of-care validated hearing and vision screening applications (hearScreen app, hearX Group, South Africa and Peek Acuity app, Peek Vision, United Kingdom) [26]. The hearScreen app is a lowcost app operable on an entry-level smartphone running Android OS software with off-the-shelf calibrated circumaural headphones that utilises pre-specified screening protocols to assess hearing using automated sequences and employs noise-monitoring algorithms for quality control [21, 23, 24, 34-37]. The peekAcuity app was designed and validated to test visual acuity proved capable of accurate and repeatable acuity measurements $[15,25]$. The use of these validated smartphone screening apps incorporating automated testing and measures of quality control allowed trained CHWs to decentralise hearing and vision screening and to identify cases for referral [21, 23-25, 27, 29, 34-37]. A detailed description of the pilot and preparation phase of the programme, the training for CHWs, screening procedures and equipment were previously described by Eksteen et al. [26, 32] . Thresholds for failing the hearing screening were set at $25 \mathrm{~dB}$ hearing level at 1,2 and $4 \mathrm{kHz}$ from September 2017 until December 2018, and $30 \mathrm{~dB}$ HL at $1 \mathrm{kHz}$ and $25 \mathrm{~dB} H \mathrm{HL}$ at 2 and $4 \mathrm{kHz}$ from January to June 2019 [32]. Children were considered to have failed the initial vision screening if they had a visual acuity of less than $0.3 \mathrm{Log}$ MAR in both eyes, or less than 0.4 LogMAR in one eye regardless of acuity in the other eye [4].

\section{Follow-up assessments}

All children who failed the screening were scheduled to undergo a follow-up assessment at their preschool.

Children who failed the hearing test received a follow-up assessment by an Audiologist at their preschool a week or two later [32]. The follow-up hearing assessment included otoscopy (Welch Allyn otoscope) and air conduction threshold pure tone audiometry using the validated hearTest app (hearX Group, South Africa) [32, 34, 35] on a Samsung A3 smartphone with the operating system Android version 8.0 (Google, United States of America), connected to supra-aural Sennheiser HD280 headphones (Sennheiser, Wedemark, Germany). Equipment had been calibrated according to prescribed standards (International Organization for Standardization, ISO 389-1). The app is calibrated to monitor environmental noise with the smartphone microphone [23, 35-37]. A warning was given when environmental noise exceeded minimal permissible ambient noise levels and the test could be paused until the noise levels were within an acceptable range [32]. Automated audiometry consisted of air conduction testing at 0.5 to $8 \mathrm{kHz}$ starting at an intensity level of $40 \mathrm{~dB} \mathrm{HL}$ until a minimum response level of $10 \mathrm{~dB} \mathrm{HL}$ [32]. The threshold determination sequence follows the Threshold Ascending method as specified in ISO 82531:1.5. As no tympanometry or bone conduction audiology was done at the follow-up assessment, cases were categorized into children with "no signs of external or middle ear abnormalities" or "obvious signs of external or middle ear abnormalities" based on the otoscopic evaluation conducted by the Audiologist. "Obvious signs of external or middle ear abnormalities" included observations of occluding wax, otorrhoea or abnormal tympanic membrane. Criteria constituting hearing loss was pure tone average (PTA) $(0.5-4 \mathrm{kHz})$ of $25 \mathrm{~dB} \mathrm{HL}$ or greater in the worse ear [8]. Degree of hearing loss was largely based on the classification by the World Health Organization (26-40 dB HL being "mild", 41-60 dB HL "moderate", 61-80 dB HL "severe" and $81 \mathrm{~dB}$ HL or greater "profound") [3]; $25 \mathrm{~dB}$ HL was included in the "mild" category.

Children who failed the initial vision screening were retested on the same day at their preschool by the trained CHWs, using the validated Peek Acuity application on the same smartphone (Peek Vision, London, United Kingdom) [26]. This test follows the standard Early Treatment Diabetic Retinopathy Study chart design, using a Tumbling E optotype, and is capable of acuity measurements consistent with test-retest variability of acuities measured using 5-letters-per line retro-illuminated LogMAR (logarithm of minimum angle of resolution) charts $[25,26]$. Vision loss was indicated when the visual acuity was less than 0.3 LogMar in both eyes, or less than 0.4 LogMar in the worse eye. Degree of vision loss was categorized as "Mild" (0.4 LogMar), "Moderate" (0.5-0.9 LogMar), "Severe" (1-4 LogMar) and "No Response" (5 LogMar) [4]. Recent studies demonstrated that minimally trained non-specialist health workers (e.g. CHWs) are able to conduct screening services equivalent to that of professional healthcare workers, when equipped with mHealth technology $[27,28,30]$.

\section{Referrals after follow-up assessments}

Children presenting with hearing or vision loss at the follow-up assessment were referred to public health care clinics (audiology or optometry clinics) in their area for further assessments and intervention [26, 32]. Children whose hearing was unable to be tested due to inconsistent and unreliable responses, were recorded on the database as "unable to test" and referred for evaluation at the health care clinics. Children who presented 


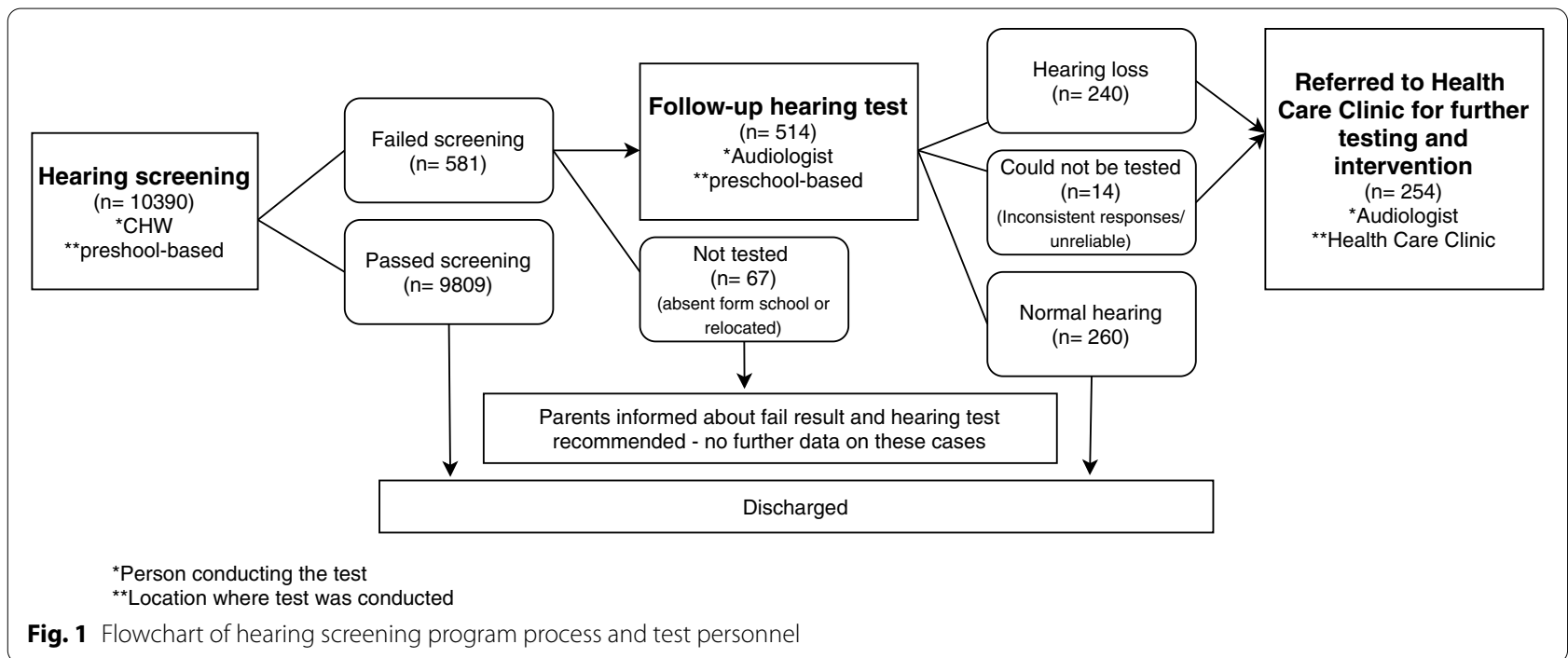

with "Normal" results (-0.1-0.3 LogMar), but had obvious signs of ocular abnormality (such as strabismus or a teacher's report of visual difficulty), were recorded on the database as "ocular morbidity" and referred for evaluation at the health care clinics. Parents of children who were referred were notified of the outcome via a letter and phone call [26]. A future study will report on the outcome of the clinic visits.

\section{Data storage and analysis}

Data collected by the smartphone were uploaded to a cloud storage facility through mobile telephone networks at the end of each test [36, 37], using the mHealth Studio platform (hearX Group, South Africa) [26, 32]. The security of the mHealth app and server are provided by local data encryption at rest using Advanced Encryption Standard 256 bit [26].

Data of the follow-up test were extracted from the secure cloud-based server (mHealth Studio) to Microsoft Excel (2016) and coded according to test outcomes (sensory loss or not), characteristics (unilateral or bilateral) and severity of loss for descriptive statistical analysis. Logistic regression was used to estimate the association between the presence of sensory loss and gender and age using IBM SPSS Statistics for Windows (version 25.0 Armonk, NY). A $p$-value cut off was set at 0.05 and indicated the level of significance throughout this study.

\section{Results}

A total of 10,390 children (50.2\% female) with a mean age of 5.7 years (SD 0.61) were screened at 298 preschools over 22 months (Figs. 1 and 2).

The overall screening referral rate was 5.6\% (581 children) resulting from hearing screening (Fig. 1) and 4.4\%
(453) resulting from vision screening (Fig. 2). Seventytwo children $(0.7 \%)$ failed both hearing and vision screening at the initial assessment.

\section{Follow-up hearing test}

Follow-up hearing tests at the preschools were done on $88.5 \%$ (514) of children of whom 240 children $(54.2 \%$ female) presented with hearing loss (Table 1 and Fig. 1). Fourteen of the children who failed the hearing screening and who were seen for a follow-up assessment, were unable to be tested due to inconsistent responses (Fig. 1). They were referred to the health care clinic for further tests, but they were not included as children with a hearing loss in this study. Half $(260,51 \%)$ of children presented with normal hearing at the follow-up hearing test and were discharged from the programme (Fig. 1). Prevalence for hearing loss at a PTA of $25 \mathrm{~dB}$ HL ranged between 2.3\% (240/10390) (assuming none of the nonattenders and "unable to test" children had hearing loss) and 3.1\% (321/10390) (assuming all the non-attenders and "unable to test" children presented with hearing loss). Of the 136 children with obvious signs of external and/or middle ear abnormalities, 95 (69.9\%) had occluding wax and 41 (30.1\%) had abnormal middle ear findings (Table 1).

\section{Follow-up vision test}

A follow-up vision test was done on 400 children $(88.1 \%)$ on the same day that they failed the initial screening at the preschool (Fig. 2). A total of 232 children $(46.1 \%$ female) had a vision impairment at the set criteria (Table 2), and a further 32 children passed the test but had obvious signs of ocular morbidity (Fig. 2). Prevalence of vision loss ranged between 2.2\% 


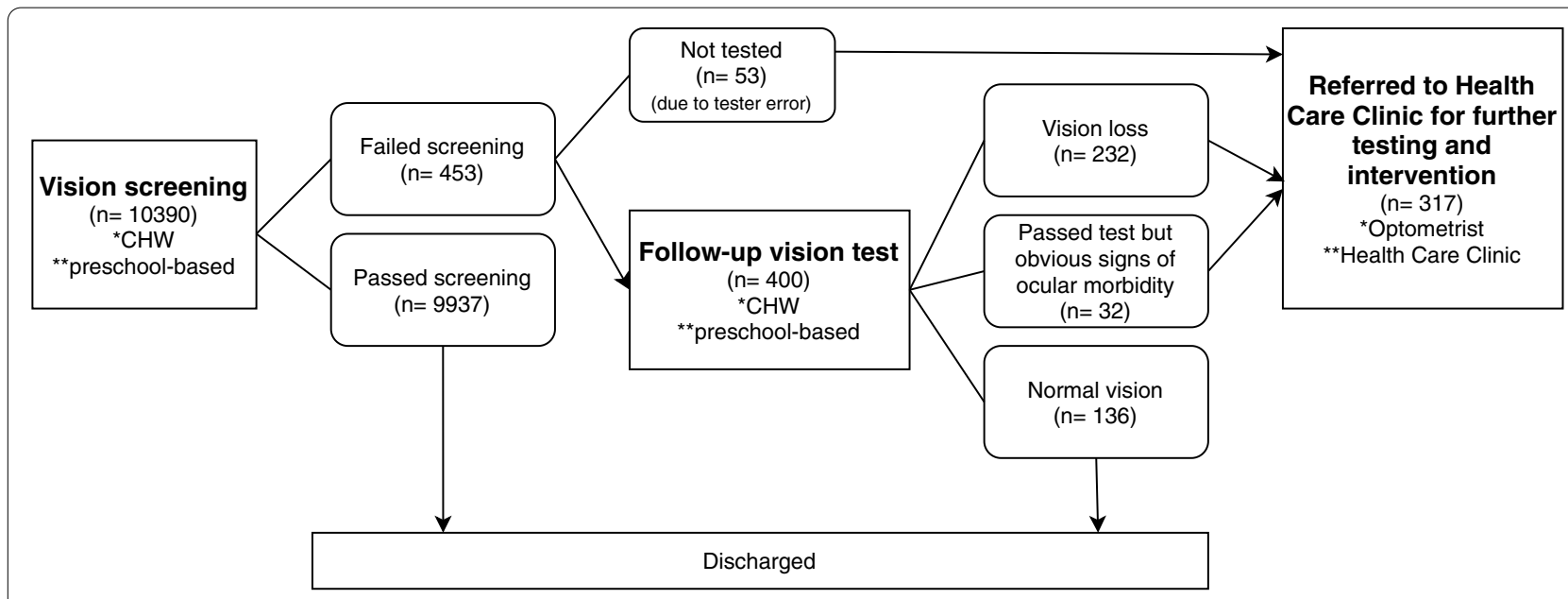

${ }^{*}$ Person conducting the test

** Location where test was conducted

Fig. 2 Flowchart of vision screening program process and test personnel

Table 1 Characteristics of hearing loss across participants seen for follow-up by audiologist at preschools $(n=240)$

\begin{tabular}{|c|c|c|c|}
\hline Characteristics & Bilateral \% (n) & Unilateral \% ( $n$ ) & All losses \% (n) \\
\hline \multicolumn{4}{|l|}{ Hearing Loss } \\
\hline No signs of external or middle ear abnormality & $64.4 \%(67 / 104)$ & $35.6 \%(37 / 104)$ & $43.3 \%(104 / 240)$ \\
\hline External or middle ear abnormality & $64.7 \%(88 / 136)$ & $35.3 \%(48 / 136)$ & $56.7 \%(136 / 240)$ \\
\hline All hearing losses & $64.6 \%(155 / 240)$ & $35.4 \%(85 / 240)$ & $100 \%(240 / 240)$ \\
\hline \multicolumn{4}{|l|}{ Degree of $\mathrm{HL}$ according to the worst ear } \\
\hline Mild (25-40 dB HL) & $63.6 \%(84 / 132)$ & $36.4 \%(48 / 132)$ & $55.0 \%(132 / 240)$ \\
\hline Moderate (41-60 dB HL) & $66.3 \%(57 / 86)$ & $33.7 \%(29 / 86)$ & $35.8 \%(86 / 240)$ \\
\hline Severe (61-80 dB HL) & $27.3 \%(3 / 11)$ & $72.7 \%(8 / 11)$ & $4.6 \%(11 / 240)$ \\
\hline Profound ( $81 \mathrm{~dB}$ HL or greater) & $100 \%(11 / 11)$ & 0 & $4.6 \%(11 / 240)$ \\
\hline
\end{tabular}

Table 2 Characteristics of vision loss across participants seen for follow-up vision test at preschools $(n=232)$

\begin{tabular}{llll}
\hline Characteristic & Bilateral \% $(\boldsymbol{n})$ & Unilateral \% (n) & All losses \% (n) \\
\hline $\begin{array}{l}\text { Vision Loss } \\
\quad \text { All vision losses }\end{array}$ & $59.1 \%(137 / 232)$ & $40.9 \%(95 / 232)$ & $100 \%(232 / 232)$ \\
Degree of VL according to the worst eye & & & $4.7 \%(11 / 232)$ \\
$\quad$ Mild (0.4 LogMar) & $100 \%(11 / 11)$ & 0 & $38.4 \%(89 / 232)$ \\
Moderate (0.5-0.9 LogMar) & $56.2 \%(50 / 89)$ & $43.8 \%(39 / 89)$ & $7.8 \%(18 / 232)$ \\
$\quad$ Severe (1-4 LogMar) & $66.7 \%(12 / 18)$ & $33.3 \%(6 / 18)$ & $49.1 \%(114 / 232)$ \\
No Response (5 LogMar) & $56.1 \%(64 / 114)$ & $43.9 \%(50 / 114)$ & \\
\end{tabular}

(232/10390) (assuming none of the non-attenders had vision loss) and $2.8 \%(286 / 10390)$ (assuming all the non-attenders presented with vision loss). The laterality and degree of vision loss is shown in Table 2.
Table 3 displays the prevalence of hearing loss and vision loss in the population of children screened at their preschools. 
Table 3 Prevalence of sensory losses in the population of children screened at preschools $(n=10,390)$

\begin{tabular}{|c|c|c|c|}
\hline Characteristics & All losses \% (n) & Bilateral \% (n) & Unilateral \% ( $n$ ) \\
\hline \multicolumn{4}{|l|}{ Hearing Loss } \\
\hline No signs of external or middle ear abnormality & $1.0 \%(104 / 10390)$ & $0.6 \%(67 / 10390)$ & $0.4 \%(37 / 10390)$ \\
\hline External or middle ear abnormality & $1.3 \%(136 / 10390)$ & $0.8 \%(88 / 10390)$ & $0.5 \%(48 / 10390)$ \\
\hline All hearing losses & $2.3 \%(240 / 10390)$ & $1.5 \%(155 / 10390)$ & $0.8 \%(85 / 10390)$ \\
\hline \multicolumn{4}{|l|}{ Degree of $\mathrm{HL}$ according to the worst ear } \\
\hline Mild (25-40 dB HL) & $1.3 \%(132 / 10390)$ & $0.8 \%(84 / 10390)$ & $0.5 \%(48 / 10390)$ \\
\hline Moderate (41-60 dB HL) & $0.8 \%(86 / 10390)$ & $0.5 \%(57 / 10390)$ & $0.3 \%(29 / 10390)$ \\
\hline Severe (61-80 dB HL) & $0.1 \%(11 / 10390)$ & $0.02 \%(3 / 10390)$ & $0.08 \%(8 / 10390)$ \\
\hline Profound (81 dB HL or greater) & $0.1 \%(11 / 10390)$ & $0.1 \%(11 / 10390)$ & 0 \\
\hline \multicolumn{4}{|l|}{ Vision Loss } \\
\hline All vision losses & $2.2 \%(232 / 10390)$ & $1.3 \%(137 / 10390)$ & $0.9 \%(95 / 10390)$ \\
\hline \multicolumn{4}{|l|}{ Degree of VL according to the worst eye } \\
\hline Mild (0.4 LogMar) & $0.1 \%(11 / 10390)$ & $0.1 \%(11 / 10390)$ & 0 \\
\hline Moderate (0.5-0.9 LogMar) & $0.9 \%(89 / 10390)$ & $0.5 \%(50 / 10390)$ & $0.4 \%(39 / 10390)$ \\
\hline Severe (1-4 LogMar) & $0.2 \%(18 / 10390)$ & $0.1 \%(12 / 10390)$ & $0.1 \%(6 / 10390)$ \\
\hline No Response (5 LogMar) & $1.1 \%(114 / 10390)$ & $0.6 \%(64 / 10390)$ & $0.5 \%(50 / 10390)$ \\
\hline
\end{tabular}

Table 4 Prevalence of sensory impairment according to age and gender

\begin{tabular}{llllll}
\hline & & $\begin{array}{l}\text { Distribution of } \\
\text { participants }(\mathbf{n})\end{array}$ & $\begin{array}{l}\text { \% of children with } \\
\text { hearing loss }(\mathbf{n})\end{array}$ & $\begin{array}{l}\text { \% of children with } \\
\text { vision loss }(\mathbf{n})\end{array}$ & $\begin{array}{l}\text { \% of children with } \\
\text { combined sensory } \\
\text { loss (n) }\end{array}$ \\
\hline Total & & $100 \%(10390)$ & $2.3 \%(240)$ & $2.2 \%(232)$ & $0.3 \%(27)$ \\
Gender & Female & $50.2 \%(5215)$ & $2.5 \%(130)$ & $2.1 \%(107)$ & $0.2 \%(12)$ \\
& Male & $49.8 \%(5175)$ & $2.1 \%(110)$ & $2.4 \%(125)$ & $0.3 \%(15)$ \\
Age & $\mathbf{4 - 5}$ years & $17.4 \%(1808)$ & $2.5 \%(45)$ & $3.7 \%(67)$ & $0.5 \%(9)$ \\
& $\mathbf{5 . 1 - 6 y e a r s}$ & $55.0 \%(5715)$ & $2.4 \%(137)$ & $2.4 \%(136)$ & $0.3 \%(17)$ \\
& $\mathbf{6 . 1 - 7 y e a r s}$ & $27.6 \%(2867)$ & $2.0 \%(58)$ & $1.0 \%(29)$ & $0.03 \%(1)$ \\
\hline
\end{tabular}

Table 4 displays the distribution of sensory losses according to age and gender in children tested at their preschool. Logistic regression analysis found that age was a significant predictor of vision loss $(p<0.001)$, with each year older a participant was $51.4 \%$ less likely of having vision loss (OR: 0.49, 95\% CI:0.39-0.60). Age was not a significant predictor of hearing loss $(p>0.05)$. Gender was not a significant predictor of hearing $(p>0.05)$ or vision loss $(\mathrm{p}>0.05)$.

\section{Discussion}

This study aimed to estimate and describe hearing and vision loss among preschool children (4-7years) in an underserved South African community. A critical issue in health services research related to infants and children is that of timely, necessary, and appropriate referrals for early childhood intervention services $[1,2,16]$. The development of mHealth has provided more opportunities for sensory screening at preschools in the community, to facilitate increased access to hearing and vision services. In this study, 5.6 and $4.4 \%$ of children failed the initial hearing and vision screen, respectively. These estimates compare well with previous studies reporting estimate referral rates of $5.6 \%$ for hearing [36] and 3.6\% for vision [24]. Despite literature reporting that hearing and vision loss commonly co-occur [38, 39], only $0.7 \%$ of children failed both hearing and vision screening, indicating the value of offering dual sensory screening at the same time, as identifying an impairment in one modality does not predispose or preclude an impairment in the other [26]. This service-delivery model, where trained CHWs are utilized to screen both hearing and vision using the same smartphone, has been shown to be efficient and low-cost [24, 26].

A high proportion of the children who failed the screens completed the follow-up assessments $(88.5 \%$ for hearing and $88.3 \%$ for vision). These figures are high compared to rates of 32.5 and $25.1 \%$ reported by Manus et al. (2020) and $45.3 \%$ reported by Hussein et al. (2018), when follow-up assessments were done 
at the health care facilities [23, 24]. Loss to followup after screening is widely reported as a barrier to healthcare $[8,23,24,27]$. In previous studies, reasons for poor follow-up rates were attributed to transportation costs, leave of absence from work and long waiting periods at health care facilities $[16,23,30]$. The high follow-up rates of this study demonstrate the value of decentralized follow-up assessments conducted at the preschools in the community $[26,40]$. In this study, the follow-up hearing tests were done by an audiologist. In low-resource settings, the availability and capacity of audiologists may pose a challenge to scaling up this model. For future implementation of such services, it is therefore proposed to enable CHWs to gather both threshold audiometric data and otoscopic images using a unified smartphone-based platform [21]. With smartphone-enabled otoscopes (smartphones coupled with specialized cameras allowing otoscopy to be utilized on the same platform), CHWs can easily capture images of the ear canal and tympanic membrane and save them to be shared and referenced in the future [21, 41]. The utilization of trained CHWs can further contribute to the affordability and the efficiency of the applied servicedelivery model $[21,24,26]$.

Out of the children who failed hearing and vision screening, $41.3 \%$ presented with hearing loss and 51.2\% presented with vision loss at the follow-up assessment and were referred for treatment in the health care system. The community-based follow-up assessments assure selective referrals, thereby reducing the burden upon the health care systems and scarce specialized healthcare professionals [19, 21, 26].

Due to the risk of loss to follow-up at health care centres, it is more accurate to report the prevalence of sensory losses according to the follow-up assessments at the preschools at that point in time [23, 24]. The prevalence for hearing loss in this study ranged between 2.3 and $3.1 \%$, depending on the assumptions for the proportion of non-respondents who were cases. Different criteria and testing methods and age cut-offs are used to determine sensory losses across studies, making it difficult to compare these prevalence estimates with the existing literature $[23,42]$. The global prevalence of disabling hearing impairment (defined as PTA $\geq 35 \mathrm{~dB}$ HL in the better ear) among children 5-14years of age was reported as $1.4 \%$ and prevalence in sub-Saharan Africa was 1.9\% [10], whereas a study by Olusanya et al. (2020) reported global prevalence in 5-9year olds for hearing loss as 3.8 and 4.5\% for sub-Saharan Africa (criteria constituting hearing loss was PTA $\geq 20 \mathrm{~dB}$ HL in the better ear) [2]. Prevalence estimates have also been reported in preschool children in sub-Saharan Africa, ranging from 2.4\% in Zimbabwe [43] and to $21.3 \%$ in Nigeria [44].
About half of the children with hearing loss (53.5\%) had obvious signs of external and/or middle ear abnormalities. The prevalence of ear disease might have been even higher, as tympanometry was not conducted and therefore not all middle ear pathology was identified $[8$, 45]. The high prevalence of occluding wax and abnormal middle ear findings in the current study are in line with recent reports from the $\mathrm{WHO}$, which postulates that the leading causes of childhood hearing loss in LMICs are conductive and treatable [46]. Studies have found conductive hearing loss to be the most common type of hearing loss found in preschool children in South Africa (65\% in both studies) [23, 47]. This indicates a need for referral services in sub-Saharan Africa in order to ensure for appropriate treatment and follow-up service and highlights ear disease as a public health concern $[22,23$, $47,48]$.

The prevalence of bilateral hearing loss was found to be more common than that of unilateral hearing loss, in agreement with reports of others [23, 47]. Also in agreement with other studies, mild hearing loss was most prevalent, followed by moderate loss [23, 47]. This may be partially explained by impacted wax and otitis media and its sequelae [2, 10, 23, 47]. Early identification and appropriate management of both bilateral and unilateral hearing loss, as well as milder degree of hearing impairment are important since even a unilateral or mild hearing losses negatively affect educational outcome $[2,22$, $46,49]$. Only $0.1 \%$ of children screened had a severe hearing loss and $0.1 \%$ of children had a profound hearing loss. In recent years, an increase of targeted hearing screening in Cape Town, South Africa, resulted in more children with permanent congenital or early-onset hearing loss (PCEHL) being identified and diagnosed at health care centres before the age of 4 years $[47,50]$. Therefore, children with sensory losses between 4 to 7 years might already be enrolled into intervention programmes and preschools specifically for children with disabilities, thus excluding them from the prevalence reported in this study.

Prevalence of vision loss in the current study ranged between 2.2 and $2.8 \%$. The global prevalence, as well as the prevalence in SSA, for 5-9year olds are estimated at $3.1 \%$ for vision loss [2]. In comparison to previous studies, mild vision loss was least prevalent [2]. The severity of vision loss was based on degree of loss in the worst eye, possible contributing to the high prevalence of results indicating "No response" in this study.

More than half (137) of children with vision loss had bilateral loss and 95 had unilateral loss. Thirty-two children passed the visual acuity assessment, but had obvious ocular abnormality. Nirmalan (2003) found that CHWs can be trained effectively to identify children with 
ocular abnormalities and they should not be limited to screening for vision impairment alone [51]. Therefore, training of the CHWs should include identification of obvious signs of visual impairment (such as strabismus), in order for children who passed screening but present with abnormalities to also be referred for follow-up assessments and intervention. In LMICs, the majority of vision loss is either preventable or treatable [12, 16]. Therefore, early identification and intervention through vision screening is a priority within the WHO VISION 2020 (Right to Sight) programme [12].

It is reported that sensory impairments commonly cooccur, with an estimated 40 to $60 \%$ of children with hearing loss also having some degree of vision loss [38, 39]. In the population of children diagnosed with PCEHL at health care centres [47], there will most probably be a higher incidence of co-occurring sensory losses than the $0.3 \%$ of children found to have combined sensory losses in this study. Another consideration is that early childhood education is not compulsory in South Africa and it is possible that not all young children with sensory deficits attended preschool facilities targeted in this study $[23,26]$.

In agreement with previous studies, gender did not have a significant effect on sensory losses [22, 23, 36]. Age was a predicting factor of vision loss, however, the strength of the correlation was poor. The higher prevalence of vision loss in younger children might be ascribed to younger children not yet being enrolled in special schools or receiving treatment elsewhere. Other studies also showed no association between hearing impairment and age $[22,23]$.

\section{Strengths and limitations}

Strengths of this study include a large study population, assessment of both hearing and vision, as well as the use of validated tools for community-based screening and assessments. Limitations of the current study included that sensitivity and specificity for these assessments could not be determined. The hearing assessment protocol did not include tympanometry or bone conduction audiometry and therefore, the nature (conductive versus sensori neural versus mixed hearing loss) and cause of hearing loss could not be determined. The visual assessment protocol did not include a basic ocular examination using torchlight and may have resulted in an underestimation of ocular morbidity. The cause of vision loss was also not determined.

\section{Conclusions}

According to this study, hearing loss is prevalent in at least 22 per 1000 and vision loss is prevalent in at least 23 per 1000 preschool children in an underserved South
African community. Community-based follow-up assessments ensured a high follow-up return rate $(88.5 \%$ for hearing and $88.3 \%$ for vision) and assured selective referrals, thereby reducing the burden upon the health care systems and scarce specialized healthcare professionals. Children who were identified with sensory losses were referred to health care clinics where they received interventions (e.g. medical management, hearing aids or spectacles). Future studies aim to report on causes of visual or hearing loss, as well as outcomes and the impact of interventions on the children diagnosed with sensory impairments. Timely identification of sensory losses is essential to ensure optimal outcomes and can be facilitated through community-based hearing and vision services supported by mHealth technology.

\section{Abbreviations}

CHWs: Community Health Workers; dB HL: Decibel hearing level; kHz: Kilohertz; LMICs: Low-and middle income countries; PCEHL: Permanent congenital early-onset hearing loss; PTA: Pure tone average; SDGs: Sustainable development goals; SSA: Sub-saharan Africa; WHO: World Health Organization.

\section{Acknowledgements}

The authors would like to acknowledge and thank the Carel du Toit Centre and Trust, as well as all participants involved with/in this study.

\section{Authors' contributions}

SE, DS, SL, HK and RE conceptualized the study. SE and DS curated the data, conducted the analysis, interpreted the results and wrote the original draft of the paper. SE, RE, HK, SL and DS substantively reviewed and wrote the final version of the paper. All authors read and approved the final manuscript.

\section{Funding}

The research project was supported financially by the Newton Advanced Fellowship Award (NP2020PA\100013)' awarded to De Wet Swanepoel and Hannah Kuper. Susan Eksteen received funding (research grant) from Sonova AG (https://www.sonova.com/en). Stefan Launer is an employee of Sonova AG. The contents of this work are solely the responsibility of the authors and do not necessarily represent the official views of the supporting organisations. The funders had no role in study design, data collection and analysis, decision to publish, or preparation of the manuscript. The authors would also like to acknowledge and thank the Hear the World Foundation for funding the community-based screening programme (https://www.hear-the-world.com/ en).

\section{Availability of data and materials}

The dataset used and analysed during the current study are available from the corresponding author on reasonable request.

\section{Declarations}

\section{Ethics approval and consent to participate}

Institutional Review Board clearance for the study was obtained from the University of Pretoria (HUM020/1019). Only children who returned informed consent letters signed by their caregivers were screened and included in the study.

\section{Consent for publication}

Not applicable.

\section{Competing interests}

The hearScreen ${ }^{\mathrm{TM}}$ and hearTest ${ }^{\mathrm{TM}}$ application is intellectual property owned, patented, and trademarked by the University of Pretoria. The product is being developed and commercialised by the hearX group (www.hearxgroup.com). 
De Wet Swanepoel's relationship with the hearX Group includes equity and consulting.

\section{Author details}

${ }^{1}$ Department of Speech-Language Pathology and Audiology, University of Pretoria, Lynnwood Rd, Hatfield, Pretoria, Gauteng, South Africa. ${ }^{2}$ Ear Science Institute Australia, Subiaco, Australia. ${ }^{3}$ Ear Sciences Centre, The University of Western Australia, Nedlands, Australia. ${ }^{4}$ International Centre for Eye Health, Clinical Research Department, London School of Hygiene and Tropical Medicine (LSHTM), London, England. ${ }^{5}$ Sonova AG, Science \& Technology, Stäfa, Switzerland. ${ }^{6}$ School of Health and Rehabilitation Science, University of Queensland, Brisbane, Australia.

\section{Received: 12 January 2021 Accepted: 23 December 2021} Published online: 05 January 2022

\section{References}

1. Global Research on Developmental Disabilities Collaborators. Developmental disabilities among children younger than 5 years in 195 countries and territories, 1990-2016: a systematic analysis for the global burden of disease study 2016. Lancet Glob Health. 2018;18:e1100-21. https://doi. org/10.1016/S2214-109X(18)30309-7.

2. Olusanya BO, Wright SM, Nair MKC, Boo N. Global burden of childhood epilepsy, intellectual disability and sensory impairments. Pediatrics. 2020;146(1):1-17. https://doi.org/10.1542/peds.2019-2623.

3. World Health Organization. (2017a). Deafness and Hearing loss. Retrieved June, 2019, from http://www.who.int/mediacentre/factsheets/fs300/en/

4. World Health Organization. (2017b). Vision Impairment and blindness Retrieved September 3, 2020, from http://www.who.int/mediacentre/ factsheets/fs282/en/

5. American Academy of Pediatrics. Eye examination in infants, children, and young adults by pediatricians. Pediatrics. 2003;111(4):902-7.

6. Rahi JS, Cable N, BCVISG. Severe visual impairment and blindness in children in the UK. Lancet. 2003;362:1359-65.

7. Stenfeldt K. Preschool hearing screening in Sweden: an evaluation of current practices and a presentation of new national guidelines. Int J Pediatr Otorhinolaryngol. 2018;110:70-5. https://doi.org/10.1016/j.ijporl.2018.04. 013.

8. Emmett SD, Robler SK, Wang N, Labrique A, Gallo JJ, Hofstetter P. Hearing Norton sound: a community randomised trial protocol to address childhood hearing loss in rural Alaska. BMJ Open. 2019;9:e023078. https://doi. org/10.1136/bmjopen-2018-023078.

9. Graydon K, Waterworth C, Miller H, Gunasekera H. Global burden of hearing impairment and ear disease. J Laryngol Otol. 2019;(133):8-25. https:// doi.org/10.1017/S0022215118001275.

10. Stevens G, Flaxman S, Brunskill E, Mascarenhas M, Mathers CD. Global and regional hearing global and regional hearing impairment prevalence: an analysis of 42 studies in 29 countries. Eur J Pub Health. 2011;23(1):146-52. https://doi.org/10.1093/eurpub/ckr176.

11. Wilson BS, Tucci DL, Merson MH, O'Donoghue GM. Global hearing health care: new findings and perspectives. Lancet. 2017;390(10111):2503-15. https://doi.org/10.1016/S0140-6736(17)31073-5.

12. Gilbert C, Foster A. Childhood blindness in the context of VISION 2020 the right to sight. Bull World Health Organ. 2001;79(3):227-32.

13. Sustainable development goals report 2018. New York: United Nations Department of Economic and Social Affairs; 2018. Available from: https:// unstats.un.org/sdgs/report/2018 [cited 2019 May 22].

14. Olusanya BO, Davis AC, Wertlieb D, Boo NY, Nair MKC, Halpern R, et al. Global Research on Developmental Disabilities Collaborators. Developmental disabilities among children younger than 5 years in 195 countries and territories, 1990-2016: a systematic analysis for the Global Burden of Disease Study 2016. Lancet Glob Health. 2018;6(10):e1100-21. https://doi. org/10.1016/S2214-109X(18)30309-7 PMID: 30172774.

15. Rono H, Bastawrous A, Maclead D, Wanjala E, Tanna GL, Weiss HA, et al. Smartphone-based screening for visual impairment in Kenyan school children: a cluster randomised controlled trial. Lancet Glob Health. 2018;6:924-32. https://doi.org/10.1016/\$2214-109X(18)30244-4.

16. Keeffe J. Childhood vision impairment. Br J Ophthalmol. 2004;88:728-9. https://doi.org/10.1136/bjo.2004.040063.
17. Harris MS, Dodson EE. Hearing health access in developing countries. Curr Opin Otolaryngol Head Neck Surg. 2017;25(5):353-8. https://doi.org/ 10.1097/MOO.0000000000000392.

18. Olusanya BO, Neumann KJ, Saunders JE. The global burden of disabling hearing impairment: a call to action. Bull World Health Organ. 2014;92(5):367-73. https://doi.org/10.2471/BLT.13.128728.

19. Bush ML, Alexander D, Noblitt B, Lester C, Shinn JB. Pediatric hearing healthcare in Kentucky's Appalachian primary care setting. J Community Health. 2015;40(4):762-8. https://doi.org/10.1007/s10900-015-9997-0.

20. HearScreen: case study by UNESCO-Pearson Initiative for Literacy. Paris: United Nations Educational, Scientific and Cultural Organization; 2017. Available from: http://unesdoc.unesco.org/images/0025/002588/25887 7E. pdf [cited 2019 May 22].

21. Jayawardena ADL, Nassiri AM, Dylan AL, et al. Community health workerbased hearing screening on a mobile platform: a scalable protocol piloted in Haiti. Laryngoscope investigative otolaryngology, 1-8. 2020. https://doi.org/10.1002/lio2.361.

22. Kumar S, Aramani A, Mathew M, Bhat M, Rao V. Prevalence of hearing impairment amongst school going children in the rural field practice area of the institution. Indian J Otolaryngol Head Neck Surg. 2019;71(s2):156771. https://doi.org/10.1007/s12070-019-01651-9.

23. Yousuf Hussein S, Swanepoel DW, Mahomed F, Biagio de Jager L. Community-based hearing screening for young children using an mHealth service-delivery model. Glob Health Action. 2018;11(1). https://doi.org/ 10.1080/16549716.2018.1467077.

24. Manus, M., van der Linde, J., Kuper, H., Olinger, R., \& Swanepoel, DW. (2020). Community-based hearing and vision screening in schools in lowincome communities using mHealth technologies. Language, Speech and Hearing services in schools. In press.

25. Bastawrous A, Rono HK, Livingstone IAT, Weiss HA, Jordan S, Kuper H, et al. Development and validation of a smartphone-based visual acuity test (peek acuity) for clinical practice and community-based fieldwork. JAMA Ophthalmology. 2015;133(8):930-7. https://doi.org/10.1001/jamao phthalmol.2015.1468.

26. Eksteen S, Launer S, Kuper H, Eikelboom RH, Bastawrous A, Swanepoel DW. Hearing and vision screening for preschool children using mobile technology, South Africa. WHO Bull. 2019;5:672-80.

27. Shinn JR, Zuniga MG, Macharia I, Reppart J, Netterville JL, Jayawardena ADL. Community health workers obtain similar results using cell-phone based hearing screening tools compared to otolaryngologists in low resourced settings. Int J Pediatr Otorhinolaryngol. 2019;127(June):109670. https://doi.org/10.1016/j.ijporl.2019.109670.

28. Dawood N, Mahomed-Asmail F, Louw C, Swanepoel DW. Mhealth hearing screening for children by non- specialist health workers in communities. Int J Audiol. 2020;0(0):1-7. https://doi.org/10.1080/14992027.2020.18297 19.

29. Swanepoel DW. eHealth technologies enable more accessible hearing care. Semin Hear. 2020;1(212):133-40.

30. Bright T, Mulwafu W, Phiri M, Ensink RJH, Smith A, Yip J. Diagnostic accuracy of non-specialist versus specialist health workers in diagnosing hearing loss and ear disease in Malawi. Trop Med Int Health. 2019;24(7):81728. https://doi.org/10.1111/tmi.13238.

31. Arinze OC, Eze BI, Ude NN, Onwubiko SN, Ezisi CN. Determinants of eye care utilization in rural South-Eastern Nigeria. J Community Health. 2015;40:881-90. https://doi.org/10.1007/s10900-015-0008-2.

32. Eksteen S, Eikelboom RH, Launer S, Kuper H, Swanepoel D. Referral criteria for preschool hearing screening in resource-constrained settings: a comparison of protocols. Language, Speech and Hearing Services in Schools. 2021;202106:1-9.

33. Statistics South Africa (2011). Key statistics city of Cape Town. Available from: http://resource.capetown.gov.za/documentcentre/Documents/ Maps\%20and\%20statistics/2011_Census_CT_Suburb_Mitchells_Plain_ Profile.pdf. Accessed 16 Aug 2020.

34. van Tonder J, Swanepoel DW, Mahomed-Asmail F, Myburgh HC, Eikelboom RH. Automated smartphone threshold audiometry: validity and time efficiency. J Am Acad Audiol. 2017;28(3):200-8. https://doi.org/10. 3766/jaaa.16002.

35. Sandström J, Swanepoel DW, Carel Myburgh H, Laurent C. Smartphone threshold audiometry in underserved primary health-care contexts. Int J Audiol. 2016;55(4):232-8. https://doi.org/10.3109/14992027.2015.11242 94. 
36. Mahomed-Asmail F, Swanepoel DW, Eikelboom RH, Myburgh HC, Hall J. Clinical validity of hearScreenTM smartphone hearing screening for school children. Ear Hear. 2016;37(1):e11-7. https://doi.org/10.1097/AUD. 0000000000000223

37. Swanepoel DW, Myburgh HC, Howe DM, Mahomed F, Eikelboom RH. Smartphone hearing screening with integrated quality control and data management. Int J Audiol. 2014;53(12):841-9. https://doi.org/10.3109/ 14992027.2014.920965.

38. Bakhshaee M, Banaee T, Ghasemi MM, Nourizadeh N, Shojaee B, Shahriari $\mathrm{S}$, et al. Ophthalmic disturbances in children with sensorineural hearing loss. Eur Arch Otorhinolaryngol. 2009;266(6):823-5. https://doi.org/10. 1007/s00405-008-0821-7.

39. Nikolopoulos TP, Lioumi D, Stamataki S, O'Donoghue GM. Evidence-Based Overview of Ophthalmic Disorders in Deaf Children: A Literature Update. J Otol Neurotol. 2006;27(2 suppl1):S1-24.

40. Zeng, Y., Han, X., Wang, D., et.al. (2020). Effect of a complex intervention to improve post-vision screening referral compliance among pre-school children in China: a cluster randomized clinical trial. EClinicalMedicine, 19, 1-7. https://doi.org/10.1016/j.eclinm.2020.100258.

41. Moshtaghi O, Sahyouni R, Haidar YM, et al. Smartphone-enabled otoscopy in Neurotology/otology. Otolaryngol Head Neck Surg. 2017;156(3):554-8. https://doi.org/10.1177/0194599816687740.

42. Mulwafu W, Kuper H, Ensink RJH. Prevalence and causes of hearing impairment in Africa. Trop Med Int Health. 2016;21 (2):158-65. https://doi. org/10.1111/tmi.12640.

43. Westerberg BD, Skowronski DM, Stewart IF, Stewart L, Bernauer M, Mudarikwa L. Prevalence of hearing loss in primary school children in Zimbabwe. Int J Pediatr Otorhinolaryngol. 2005;69:517-25. https://doi. org/10.1016/j.jiporl.2004.11.020

44. Adebola SO, Ayodele SO, Oyelakin OA, Babarinde JA, Adebola OE. Preschool hearing screening: profile of children from Ogbomoso, Nigeria. Int J Pediatr Otorhinolaryngol. 2013;(77):1987-91. https://doi.org/10.1016/j. ijporl.2013.09.019.

45. New Zealand Health Techology Assessment. Screening programmes for the detection of otitis media with effusion and conductive hearing loss in pre-school and new entrant school children: a critical approasial of the Ilterature. Christchurch: New Zealand Health Technology Assessment Clearing House; 1998.

46. World Health Organization. (2018). Global Estimates on hearing loss. Retrieved June 26, 2020, from https://www.who.int/pbd/deafness/estim ates/en/

47. Kuschke S, Swanepoel DW, le Roux T, Strauss S. Profile of childhood hearing loss in the Western cape, South Africa. Int J Pediatr Otorhinolaryngol. 2020;137:110248. https://doi.org/10.1016/j.ijporl.2020.110248.

48. Olusanya BO, Luxon LM, Wirz SL. Benefits and challenges of newborn hearing screening for developing countries. Int J Pediatr Otorhinolaryngol. 2004;68(3):287-305. https://doi.org/10.1016/j.jporl.2003.10.015.

49. Dodd-Murphy J, Murphy W, Bess FH. Accuracy of school screenings in the identification of minimal sensorineural hearing loss. Am J Audiol. 2014;23:365-73. https://doi.org/10.1044/2014.

50. de Kock T, Swanepoel DW, Hall JW. Newborn hearing screening at a community-based obstetric unit: screening and diagnostic outcomes. Int J Pediatr Otorhinolaryngol. 2016;84:124-31. https://doi.org/10.1016/j. ijporl.2016.02.031.

51. Nirmalan PK, Vijayalakshmi P, Sheeladevi S, Kothari MB, Sundaresan K, Rahmathullah L. The Kariapatti pediatric eye evaluation project: baseline ophthalmic data of children aged 15 years or younger in southern India Am J Ophthalmol. 2003:703-9. https://doi.org/10.1016/50002-9394(03) 00421-5.

\section{Publisher's Note}

Springer Nature remains neutral with regard to jurisdictional claims in published maps and institutional affiliations. 